\title{
IS THE CALDWELL-LUC OPERATION AND ITS MODIFICATIONS STILL USEFUL IN THE ERA OF ENDOSCOPIC SINUS SURGERY? FINDINGS FROM A RESOURCE-LIMITED SETTING
}

\author{
Rowland Agbara', Benjamin Fomete', Kelvin U. Omeje ${ }^{3}$, Polycarp Onyebuchi ${ }^{2}$ \\ 'Department of Oral and Maxillofacial Surgery, Faculty of Dental Sciences, College of Health Sciences, University of Jos, Nigeria \\ ${ }^{2}$ Department of Maxillofacial Surgery, Ahmadu Bello University Teaching Hospital, Nigeria \\ ${ }^{3}$ Department of Maxillofacial Surgery, Aminu Kano Teaching Hospital, Nigeria
}

\begin{abstract}
INTRODUCTION: Despite the advent of endoscopic sinus surgery, an external approach to the maxillary sinus may be ideal in certain situations.

ОвJеCTIVES: This retrospective study reviews the relevance of, and the indications for, an external approach to the maxillary sinus via Caldwell-Luc operation or its modifications in a health resource-limited setting.

MATERIAL AND METHODS: Data from case notes and operating records of patients who had the Caldwell-Luc operation or its modifications between January 2004 and June 2018 at the Department of Maxillofacial Surgery of a University Teaching Hospital were retrospectively studied. Findings from descriptive statistics are represented in the form of tables and charts.

RESUlTs: A total of 26 patients had the Caldwell-Luc operation within the period reviewed, and this consisted of $16(61.5 \%)$ males and $10(38.5 \%)$ females, giving a male to female ratio of $1.6: 1$. Patients' ages ranged from 10 to 45 years with a mean of $31.0 \pm 8.8$ years. The paediatric age group accounted for only $11.5 \%$ of cases. The main indication for performing the Caldwell-Luc operation was chronic maxillary sinusitis $(n=14 ; 53.8 \%)$, and occipitomental radiograph $(n=24 ; 92.3 \%)$ was the main imaging modality used for assessment. Compliance with follow-up review was poor.

Conclusions: Despite the advent of functional endoscopic sinus surgery, an external approach to the maxillary sinus using the Caldwell-Luc operation is still relevant, especially in health resource-limited settings.
\end{abstract}

KEY wORDS: maxillary sinus, sinusitis, health resources, external approach.

J Stoma 2019; 72, 2: 63-69

DOI: https://

\section{INTRODUCTION}

The paired maxillary sinus in an adult is a pyramidal shaped, air-filled cavity located within the maxilla, in the middle-third of the facial skeleton. It has medial, superior (roof), lateral, anterior, posterior, and inferior (floor) walls with an apex that is directed towards the zygoma. Embryologically, the maxillary sinus develops in the third month of foetal development as invaginations of the nasal mucosa of the middle meatus into the max-

JOURNAL OF STOMATOLOGY CZASOPISMO STOMATOLOGICZNE

AdDRESS FOR CORRESPONDENCE: Rowland Agbara, Department of Oral and Maxillofacial Surgery, Faculty of Dental Sciences, College of Health Sciences, University of Jos, Nigeria, e-mail: row_prof@yahoo.com

ReCEIVED: 14.03.2019 • ACCEPTED: 30.05.2019 • Published: 19.07.2019 
illary bones [23]. It is rudimentary at birth and reaches adult size at puberty following the eruption of permanent teeth, with a mean volume of $12.5 \mathrm{ml}$ [8]. It may occasionally be absent or hypoplastic [3]. The functions of the maxillary sinus are controversial, but it is said to include reduction in weight of the craniofacial bones, acts as a shock absorber to traumatic forces, thermal insulation, humidification, and warming of inspired air, and influences facial growth [25]. It receives blood supply mostly from the facial, maxillary, infraorbital, and greater palatine arteries, while venous drainage is to the facial vein and pterygoid plexus. Lymphatic drainage is to the submandibular nodes and its nerve supply is by the maxillary nerve via its superior alveolar, the greater palatine, and infraorbital nerves [15].

The maxillary sinus is related to important structures such as the orbit, roots of teeth and their supplying neurovascular bundle, the nasal cavity, the palate, maxillary/ zygomatic bone, infraorbital nerve, lacrimal duct, etc. Therefore, pathologies affecting the maxillary antrum may involve these related structures. Similarly, pathologies affecting these associated structures may eventually involve the maxillary antrum. Pathologies involving the maxillary sinus may be infective (odontogenic and non-odontogenic), neoplastic, cystic, or foreign body lodgement such as teeth and bullets/pellets [2].

The maxillary antrum may be accessed surgically using several approaches for the purpose of treatment of antral pathologies or pathologies outside the maxillary antrum such as in the transantral approach to the sphenoid, infratemporal/pterygopalatine fossae, etc. Approaches to the maxillary antrum are generally classified as external, endoscopic, or a combination of both. External approaches include canine fossa puncture, transalveolar approach, Caldwell-Luc operation and its modifications, lateral antrotomy approach with or without bone flap, lateral rhinotomy approach, and intranasal approach $[4,10]$. The external and endoscopic approaches have their respective indications and contraindications with varying advantages/disadvantages [2, 4].

\section{OBJECTIVES}

Despite advances in sinus surgery using the endoscopic approach, this technique is not widely available in health resource-limited settings due to limited facilities and expertise. Hence, the external approach has enabled patients to receive treatment. This retrospective study reviews the indications for an external approach to the maxillary sinus via the Caldwell-Luc operation or its modifications (CLOM) in a health resource-challenged setting.

\section{MATERIAL AND METHODS}

Data from case notes and operating records of patients who had Caldwell-Luc operation or its modifications between January 2004 and June 2018 at the Department of Maxillofacial Surgery of a regional University Teaching Hospital were retrospectively studied. To use this data, the institutional Ethics Committee approval was not required. Patient's demographics, indication for procedure, preoperative imaging technique used, and nature of surgical procedure were retrieved and analysed using Microsoft Excel 2007 (Microsoft, Redmond, WA, USA) and Statistical Product Service Solutions (SPSS) version 16 (SPSS Inc., Chicago, IL, USA). Findings from descriptive statistics were represented in the form of tables and charts.

\section{SURGICAL PROCEDURE FOR THE CALDWELL-LUC OPERATION}

Under general anaesthesia and with the patient in a supine position, the mucogingival tissues from the central incisor to the second molar on the same side are infiltrated with diluted adrenaline solution to aid in haemorrhage control. An incision is then made using a number 15 blade from the lateral incisor to the second premolar above the root of the canine, and the mucoperiosteal tissues are reflected superiorly and inferiorly using a periosteal elevator to expose the anterior surface of the maxilla. With a round bur on a slow handpiece (or using a small chisel with a mallet), an opening is created into the maxillary antrum via the anterior surface of the maxilla superiolaterally to the canine (between the canine and the first premolar). The space is gradually widened using a bone rongeur until the antrum can be visualised or the required instruments admitted. In maxillary sinusitis, all diseased mucosa are removed and the cavity irrigated with sterile normal saline solution, packed with ribbon gauze impregnated with antibiotic, and exited via an inferior nasal antrostomy performed at a point posterior-inferior to the natural ostium to avoid damage to the nasolacrimal duct, which lies approximately $1 \mathrm{~cm}$ anterior-inferior to the natural ostium. For foreign body removal, a similar procedure is performed with/without removal of sinus mucosa or nasal antrostomy, depending on findings. Following completion of the procedure, the oral mucoperiosteal flap is sutured using $3 / 0$ resorbable suture.

\section{RESULTS}

\section{AGE AND SEX DISTRIBUTION}

A total of 26 patients had the CLOM performed within the period reviewed, and this consisted of 16 (61.5\%) males and 10 (38.5\%) females, giving a male to female ratio of $1.6: 1$. Patients' ages ranged from 10 to 45 years with a mean of $31.0 \pm 8.8$ years. Adults constituted $88.5 \%$ of patients, while the paediatric age group accounted for $11.5 \%$ of cases. 


\section{IMAGING AND INDICATIONS FOR SURGERY}

The main indication for CLOM was chronic maxillary sinusitis $(n=14 ; 53.8 \%)$, followed by maxillary tumour $(n=6 ; 23.1 \%)$ (Table 1$)$. Only a single case of maxillary cyst was treated using modified Caldwell-Luc operation (Figure 1). In one of the two cases of foreign body impaction (Figure 2) treated, the procedure was undertaken to allow for possible haemorrhage control on extrication of the impaled foreign body. Plain radiography (Figure 3 ) in the form of occipitomental radiograph $(n=24 ; 92.3 \%)$ was the commonest imaging modality used for preoperative assessment.

\section{ANAESTHESIA, SURGICAL PROCEDURE, AND FOLLOW-UP}

Local anaesthetic technique was used in two (7.7\%) cases of foreign body (tooth in antrum), while the remaining 24 (92.3\%) cases were performed under general anaesthesia. Unilateral CLOM $(n=18 ; 69.2 \%)$ was more frequently performed than bilateral procedure $(n=6$; $27.1 \%$ ). In two cases the site of the procedure was not documented. Intranasal antrostomy was additionally required in $16(61.5 \%)$ patients, and these were mainly in cases with chronic maxillary sinusitis. One patient with histological diagnosis of benign fibrous histiocytoma following biopsy was referred to another centre on request. Although 10 (38.5\%) patients presented for review, only two $(7.7 \%)$ patients had follow-up review up to a period of 2-3 years, and within this period one patient who had foreign body (shrapnel) (Figure 4) removal developed chronic maxillary sinusitis requiring surgical intervention.

\section{DISCUSSION}

Following the description of an access to the maxillary antrum by George Caldwell and Henri Luc more

TABLE 1. Patients characteristics and treatment

\begin{tabular}{|c|c|c|c|}
\hline Age & Sex & Indication & Procedure \\
\hline 16 & M & Chronic maxillary sinusitis & Bilateral CLA with nasal antrostomy \\
\hline 38 & M & Chronic maxillary sinusitis & Unilateral (RT) CLA with nasal antrostomy \\
\hline 28 & M & Chronic maxillary sinusitis & Bilateral CLA with nasal antrostomy \\
\hline 35 & $\mathrm{~F}$ & Chronic maxillary sinusitis & Bilateral CLA with nasal antrostomy \\
\hline 41 & M & Chronic maxillary sinusitis & Unilateral (LT) CLA with nasal antrostomy \\
\hline 20 & M & Maxillary Tumour & Biopsy via Unilateral CLA \\
\hline 32 & M & Chronic maxillary sinusitis & Bilateral CLA with nasal antrostomy \\
\hline 35 & M & Chronic maxillary sinusitis & CLA with nasal antrostomy \\
\hline 30 & $\mathrm{~F}$ & Chronic maxillary sinusitis & Bilateral CLA with nasal antrostomy \\
\hline 16 & $\mathrm{~F}$ & Maxillary Tumour & Biopsy via Unilateral CLA \\
\hline 31 & $\mathrm{~F}$ & Chronic maxillary sinusitis & Bilateral CLA with nasal antrostomy \\
\hline 38 & $\mathrm{~F}$ & Chronic maxillary sinusitis & CLA with nasal antrostomy \\
\hline 20 & M & Foreign body (Shrapnel) & Unilateral (RT) CLA \\
\hline 37 & M & Maxillary Tumour & Biopsy via Unilateral (LT) CLA \\
\hline 35 & $\mathrm{~F}$ & Maxillary Tumour & Biopsy via Unilateral CLA \\
\hline 36 & M & Maxillary Tumour & Biopsy via Unilateral CLA \\
\hline 10 & $\mathrm{~F}$ & Cystic maxillary lesion & Enucleation via RT CLA with nasal antrostomy \\
\hline 35 & M & Foreign body (Knife edge) & Unilateral (RT) CLA \\
\hline 27 & M & Displaced tooth into antrum & Unilateral (LT) CLA \\
\hline 35 & $\mathrm{~F}$ & Displaced tooth into antrum & Unilateral (RT) CLA \\
\hline 42 & M & Foreign body (shrapnel) & Unilateral CLA with nasal antrostomy \\
\hline 27 & M & Chronic maxillary sinusitis & Unilateral CLA with nasal antrostomy \\
\hline 25 & M & Chronic maxillary sinusitis & Unilateral CLA with nasal antrostomy \\
\hline 45 & $\mathrm{~F}$ & Chronic maxillary sinusitis & Unilateral CLA with nasal antrostomy \\
\hline 34 & M & Chronic maxillary sinusitis & Unilateral CLA with nasal antrostomy \\
\hline 37 & $\mathrm{~F}$ & Maxillary tumour & Biopsy via unilateral CLA \\
\hline
\end{tabular}

OMV - occipitomental view, LT - left, CLA - Caldwell-Luc antrostomy, CT - computed tomography, RT - right 

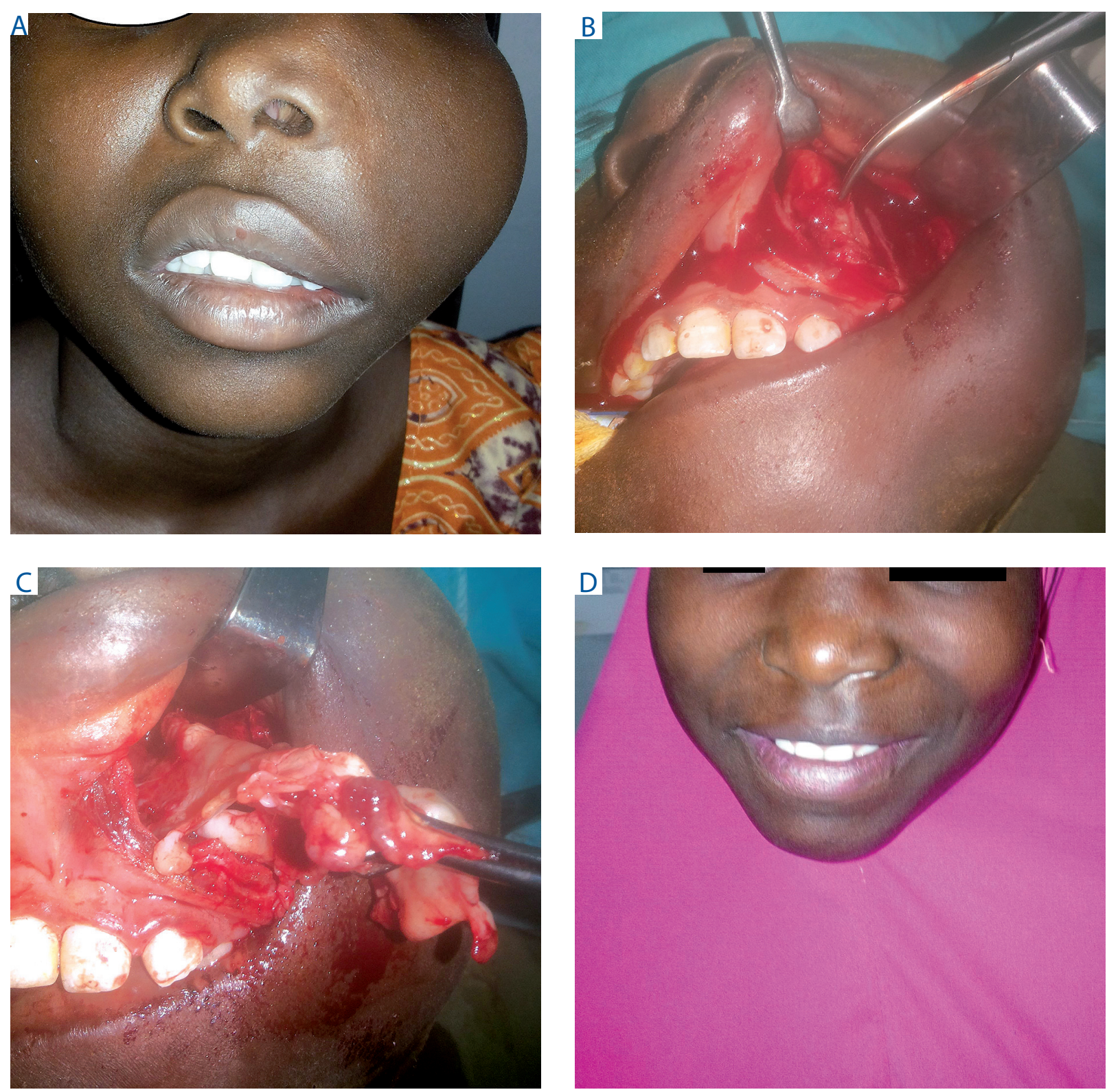

FIGURE 1. A) Facial swelling from a maxillary cyst. B) Intraoperative exposure via Caldwell-Luc approach. C) Removal of cystic lining. D) Patient review at 2 years

than 100 years ago [2], this procedure is still valuable despite the introduction of functional endoscopic sinus surgery. It is performed by oral/maxillofacial surgeons, otorhinolaryngologists, and other head/neck surgeons for various indications. The Caldwell-Luc operation in its classical form involves making an opening in the anterior wall of the maxilla, superolaterally to the canine bulge with stripping from the inner antral wall of any diseased mucosa, combined with an opening in the lateral wall of the nose inferior to the inferior turbinates. Therefore, it is essentially an intraoral antrostomy with an inferior meatal antrostomy. However, in certain cases such as in the immediate removal of a dislodged tooth in the antrum, a modified Caldwell-Luc operation is used and may involve only an intraoral antrostomy without an inferior meatal antrostomy or stripping of antral mucosa [11].

In this retrospective analysis, more males than females had CLOM, similarly to previous findings [2, 7]. However, females accounted for the major population of patients who had the Caldwell-Luc operation performed in the report by Defrietas and Lucente [6]. The male to female ratio appears to be influenced by the surgical indication for CLOM because some disease conditions usually have gender predilection. However, most antral pathologies generally appear to be more common in males [7].

The patients' ages showed an adult predominance over the paediatric population, and this is consistent with previous findings $[2,6]$. Although endonasal sinus 

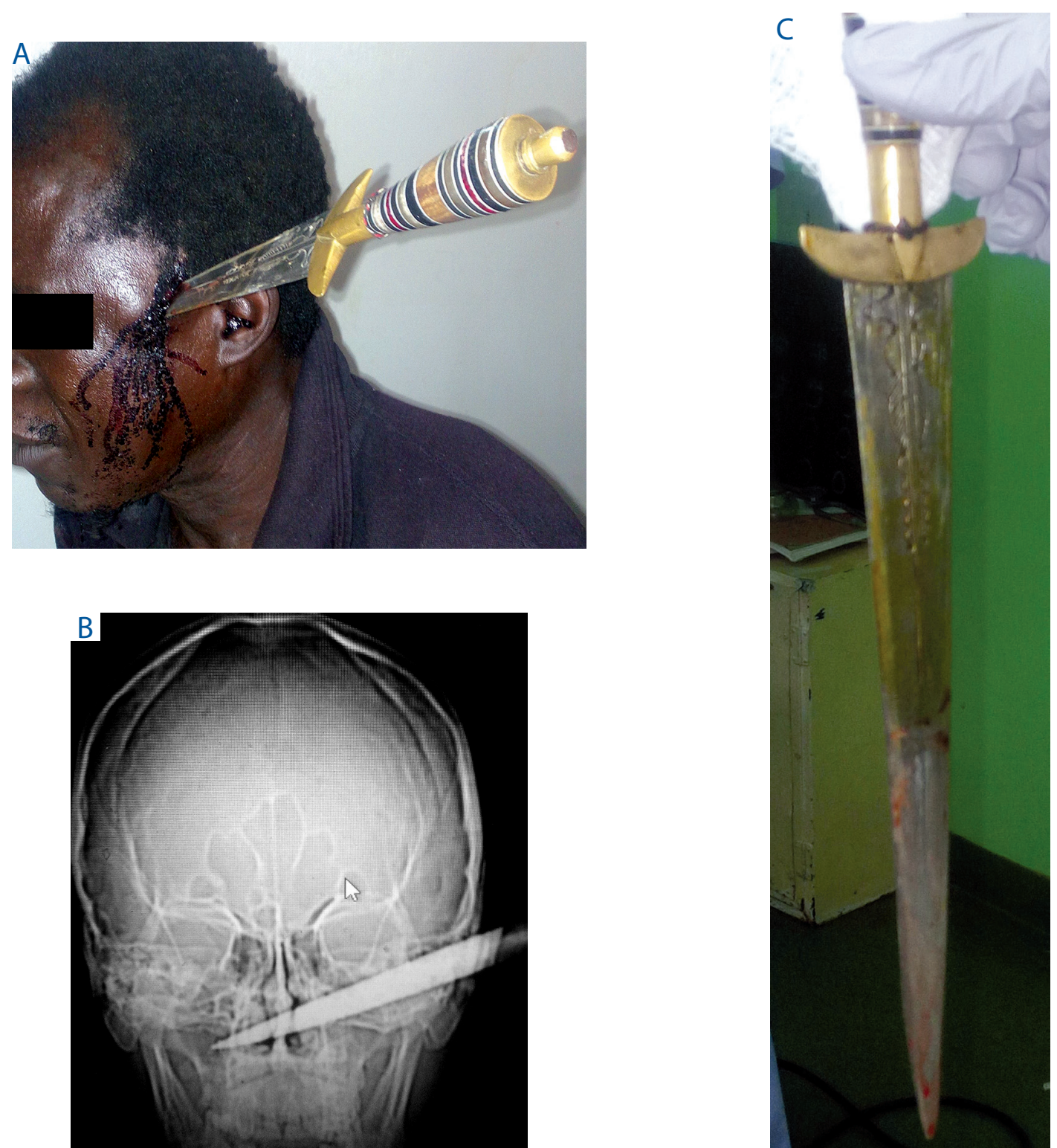

FIGURE 2. A) Impaled foreign body following assault. B) Computed tomography scanogram showing impaled foreign body extending to the right antrum. C) Removed foreign body following Caldwell-Luc approach for haemorrhage control

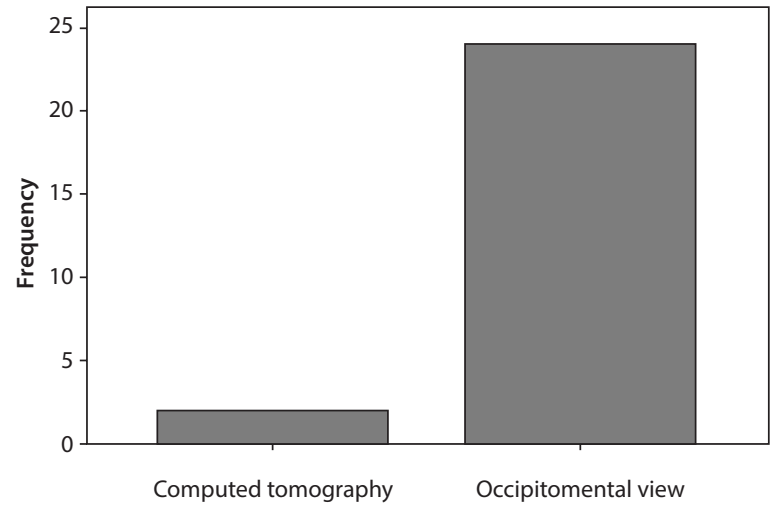

Imaging modality

FIGURE 3. Imaging modalities used in assessing patients

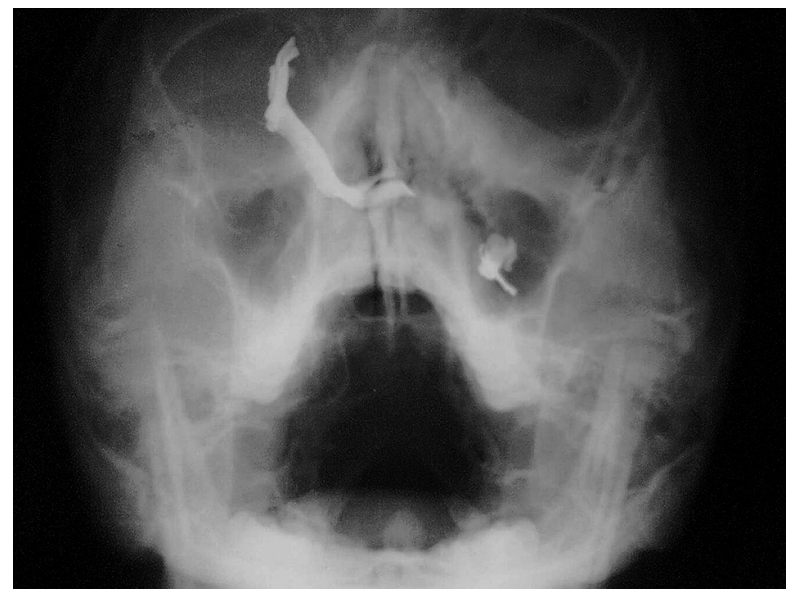

FIGURE 4. Impaled foreign body in the maxillary antrum secondary to blast injury from an improvised explosive device 
surgery has been advocated as the preferred option in the surgical treatment of chronic maxillary sinusitis in children, the Caldwell-Luc operation has been shown also to be beneficial and associated with less injury to the erupting dentition [20].

The main indication for CLOM was maxillary sinusitis followed by maxillary neoplastic lesions. This dominance of maxillary sinusitis over the other surgical indications is consistent with previous studies $[2,6]$. Over the years, the main indications for CLOM have differed among studies and include treatment of orbital floor fractures, treatment of upper jaw cystic lesions (such as dentigerous cyst) with antral extensions, foreign body removal, maxillary antral tumour biopsy, sinus augmentation, control of severe haemorrhage following facial trauma, endoscopic treatment of antral tumours, and as an access to the sphenoid and pterygomaxillary fossa [6, $9,11]$. In the present study, modified Caldwell-Luc operation was carried out in one of the patients as a ready access for haemorrhage control in the event of withdrawal of an impaled assault weapon (knife), which extended into the maxillary antrum via its posteromedial wall on computed tomography scan. On withdrawal of the impaled object, bleeding was controlled using an antral pack (ribbon gauze impregnated with antibiotic paste) and exited through an inferior meatal antrostomy.

Sinusitis, the inflammation of one or more of the paranasal sinuses, may be classified as acute (infection of the paranasal sinuses, with accompanying symptoms present for more than 10 days and less than four weeks), subacute (symptoms lasting four to eight weeks), chronic (symptoms lasting longer than eight weeks), and recurrent (presence of three or more acute episodes a year). Both acute and chronic sinusitis may require surgical treatment [16]. No patient in this study had CLOM for acute maxillary sinusitis. Maxillary sinusitis may be of odontogenic or non-odontogenic causes, with the latter accounting for $30-40 \%$ of cases in a study [21]. Odontogenic maxillary sinusitis differs in its pathophysiology, diagnosis, and management from sinusitis of other causes [26] and may arise from upper lateral or apical periodontal infections, infected odontogenic cysts, oroantral communications/fistula, dental/zygomatic implant placement, dislodged tooth or tooth fragment into the antrum, and maxillary fractures. The aetiological basis for maxillary sinusitis in the patients studied was not clearly highlighted, and therefore the role of odontogenic infection could not be established.

Various accesses to the maxillary antrum exist, and these include endoscopic, external, and combined approaches. External approaches include canine fossa puncture (which may be used in maxillary sinus lavage or combined with endoscopic approach), transalveolar approach (useful for removal of a dislodged tooth, and for sinus augmentation), Caldwell-Luc approach and its modifications, lateral antrotomy approach with or without bone flap (useful in zygoma implant placement), lateral rhinotomy approach (useful in medial maxillectomy), and intranasal approach $[4,10,18]$. The approach used will be influenced by the indication, site of the lesion, surgeon's skills, availability of equipment, etc. The endoscopic approach to the maxillary sinus commonly involves the use of rigid endoscopes (diameter $2.7 \mathrm{~mm}$ for paediatric and $4 \mathrm{~mm}$ for adults) with varying angles of vision ranging from 0 to 120 degrees [22]. Combined external and endoscopic approaches have also been used [29]. Studies comparing the Caldwell-Luc operation and endoscopic approach have shown better cure rates of sinusitis as well as markedly reduced post-operative sequelae, blood loss, operating time, and hospital stay in patients treated using functional endoscopic sinus surgery (FESS), although the Caldwell-Luc operation was associated with improved symptoms [12]. However, a higher reoperation rate has been observed in patients treated with FESS compared to those treated with Caldwell-Luc operation [19]. Despite the superior results reported with FESS, CLOM still has its indications, which include failed endoscopic middle meatal antrostomy and irreversible mucosal changes [5]. In addition, CLOM may be indicated where facilities or skills for FESS are not available, such as in resource-limited settings.

Imaging for maxillary sinus pathologies may involve the use of ultrasonography, plain radiography, or more advanced imaging techniques such as computed tomography. Ultrasonography has the advantage of been devoid of ionising radiation, it is cheap, easy to use, and can be performed by the bedside. When compared to CT in the diagnosis of paranasal diseases, ultrasonography has a sensitivity of $66.7 \%$ and a specificity of $94.9 \%$, while its sensitivity and specificity in comparison to magnetic resonance imaging (MRI) are 64\% and 95\%, respectively [14, 24]. Commonly used plain radiographs include panoramic and Waters' view. For inflammatory disease of the maxillary sinus, plain radiographs have a sensitivity of $80 \%$ [1]. Although panoramic radiographs provide information on the maxillary sinus, there is a moderate risk of false diagnosis when used in isolation. Therefore, computed tomography is recommended and considered the standard for a more precise evaluation of the maxillary sinus [17]. MRI is useful in assessing extension of paranasal sinus disease into adjacent soft tissues. Generally, three-dimensional imaging techniques compared to two-dimensional techniques have been shown to be significantly more reliable in detecting pathologies within the maxillary sinus [28]. Most of the patients in this study were evaluated using plain radiography (Water's view), only two patients had a CT scan done. The common use of plain radiography is primarily related to the inability of patients in this setting to afford the cost of a CT scan because most of them pay out of pocket for most procedures due to a poorly implemented national health insurance scheme.

CLOM may be performed under local or general anaesthesia [13], the choice of anaesthesia being dependent on patient age and tolerance, extent of proce- 
dure, surgeon's preference, and presence of co-morbid conditions, among other factors. Most of the patients in the present study had their procedure done under general anaesthesia, and this may be due to the extent of the procedures. General anaesthesia permits adequate retraction of tissues, which may not be tolerated under local anaesthesia, and permits additional procedures to be undertaken intraoperatively when the need arises.

The follow-up review of patients treated was generally poor, and therefore objective assessment of outcome was difficult. Poor response to follow-up review has consistently remained a challenge in this environment, and factors such as poverty, feeling of wellbeing, and cultural influences play a significant role. However, two patients were reviewed over 2-3 years, and of these, one patient who had foreign body (shrapnel) removal developed chronic sinusitis requiring surgical intervention. CLOM has commonly been associated with facial oedema, pain, and/or numbness of the face, teeth, and gums. Rare complications reported include epistaxis, oroantral fistula, epiphora, and tooth discolouration. ${ }^{27}$

\section{CONCLUSIONS}

Despite the advent of functional endoscopic sinus surgery, an external approach to the maxillary sinus using the Caldwell-Luc operation or its modifications is still relevant, especially in health resource-limited settings. However, poor response to follow-up in this environment does not allow for an objective assessment of outcomes relative to other studies.

\section{CONFLICT OF INTEREST}

The authors declare no potential conflicts of interest with respect to the research, authorship, and/or publication of this article.

\section{References}

1. Aaløkken TM, Hagtvedt T, Dalen I, Kolbenstvedt A. Conventional sinus radiography compared with CT in the diagnosis of acute sinusitis. Dentomaxillofac Radiol 2003; 32: 60-62.

2. Barzilai G, Greenberg E, Uri N. Indications for the Caldwell-Luc approach in the endoscopic era. Otolaryngol Head Neck Surg 2005; 132: 219-220.

3. Celebi S, Taşkın U, Altın F, Ozkul MH. Bilateral aplasia of paranasal sinuses. Eur Arch Otorhinolaryngol 2012; 269: 1055-1057.

4. Chrcanovic BR, Custódio ALN. Surgical removal of dental implants displaced into the maxillary sinus. Serbian Dental Journal 2009; 6: 139-147.

5. Cutler JL, Duncavage JA, Matheny K, Cross JL, Miman MC, Oh CK. Results of Caldwell-Luc after failed endoscopic middle meatus antrostomy in patients with chronic sinusitis. Laryngoscope 2003; 113: 2148-2150.

6. Defrietas J, Lucente FE. Caldwell-Luc procedure: institutional review of 670 cases: 1975-1985. Laryngoscope 1988; 98: 1297-1300.

7. Drumond JPN, Allegro BB, Novo NF, Miranda SL, Sendyk WR. Evaluation of the prevalence of maxillary sinuses abnormalities through spiral computed tomography (CT). Int Arch Otorhinolaryngol 2017; 21: 126-133.

8. Gosau M, Rink D, Driemel O, Draenert FG. Maxillary sinus anatomy: a Cadaveric Study with clinical implications. Anat Rec 2009; 292: 352-354.

9. Gray LN, Kalimuthu R, Jayaram B, Lewis N, Sohaey M. A retrospective study of treatment of orbital floor fractures with the maxillary sinus approach. Br J Plast Surg 1985; 38: 113-115.

10. Hong SL, Mun SJ, Cho KS, MD, Roh HJ. Inverted papilloma of the maxillary sinus: Surgical approach and long-term results. Am J Rhinol Allergy 2015; 29: 441-444.

11. Huang YC, Chen WH. Caldwell-Luc operation without inferior meatal antrostomy: a retrospective study of 50 cases. J Oral Maxillofacial Surg 2012; 70: 2080-2084.

12. Ikeda K, Hirano K, Oshima T, Takasaka T. Comparison of complications between endoscopic sinus surgery and Caldwell-Luc operation. Tohoku J Exp Med 1996; 180: 27-31.

13. Jacob KJ, George S, Preethi S, Arunraj VS. A comparative study between endoscopic middle meatal antrostomy and Caldwell-Luc surgery in the treatment of chronic maxillary sinusitis. Indian J Otolaryngol Head Neck Surg 2011; 63: 214-219.

14. Karantanas AH, Sandris V. Maxillary sinus inflammatory disease: ultrasound compared to computed tomography. Comput Med Imaging Graph 1997; 21: 233-241.

15. Last RJ. Paranasal sinuses. In: McMinn RMH (ed.). Last's Anatomy Regional and Applied. Edinburgh: Churchill Livingstone; 1994; p. 474-475.

16. Leung RS, Katial R. The diagnosis and management of acute and chronic sinusitis. Prim Care Clin Office Pract 2008; 35: 11-24.

17. Malina-Altzinger J, Damerau G, Grätz KW, Stadlinger B. Evaluation of the maxillary sinus in panoramic radiography - a comparative study. Int J Implant Dent 2015; 1: 17.

18. Morrissey DK, Wormald PJ, Psaltis AJ. Prelacrimal approach to the maxillary sinus. Int Forum Allergy Rhinol 2016; 6: 214-218.

19. NärkMäkelä M, Qvarnberg Y. Endoscopic sinus surgery or Caldwell-Luc operation in the treatment of chronic and recurrent maxillary sinusitis. Acta Otolaryngol 1997; 529: 177-180.

20. Paavolainen M, Paavolainen R, Tarkkanen J. Influence of Caldwell-Luc operation on developing permanent teeth. Laryngoscope 1977; 87: 613-620.

21. Patel NA, Ferguson BJ. Odontogenic sinusitis: an ancient but under-appreciated cause of maxillary sinusitis. Curr Opin Otolaryngol Head Neck Surg 2012; 20: 24-28.

22. Pedroletti F, Johnson BS, McCain JP. Endoscopic techniques in oral and maxillofacial surgery. Oral Maxillofacial Surg Clin N Am 2010; 22: 169-182.

23. Pikos MA. Maxillary sinus membrane repair: update on technique for large and complete perforations. Implant Dent 2008; 17: 24-31.

24. Puhakka T, Heikkinen T, Mäkelä MJ, Alanen A, Kallio T, Korsoff L, et al. Validity of ultrasonography in diagnosis of acute maxillary sinusitis. Arch Otolaryngol Head Neck Surg 2000; 126: 1482-1486.

25. Ritter FN, Lee D. The Paranasal Sinuses, Anatomy and Surgical Technique. St. Louis: The Mosby Company; 1978. p. 6-16.

26. Simuntis R, Kubilius R, Vaitkus S. Odontogenic maxillary sinusitis: a review. Stomatologija 2014; 16: 39-43.

27. Stefánsson P, Andreásson L, Jannert M. Caldwell-Luc operation: long term results and sequelaes. Acta Otolaryngol 2009; 105: 97-100.

28. Tadinada A, Fung K, Thacker S, Mahdian M, Jadhav A, Schincaglia GP. Radiographic evaluation of the maxillary sinus prior to dental implant therapy: a comparison between two-dimensional and three-dimensional radiographic imaging. Imaging Sci Dent 2015; 45: 169-174.

29. Wu X, Li A, Tan J, Ou X. Removal of orbital-maxillary sinus-pterygopalatine fossa foreign body with external and endoscopic combined approach. J Craniofac Surg 2014; 25: 1547-1549. 